\title{
Correlates and Moderators of Change in Subjective Memory and Memory Performance: Findings from the Health and Retirement Study
}

\author{
Gizem Hülür ${ }^{a}$ Christopher Hertzog ${ }^{b}$ Ann M. Pearman ${ }^{b}$ Denis Gerstorfa

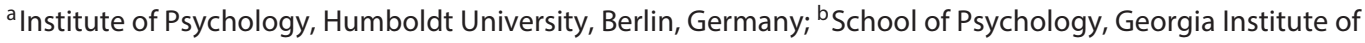 \\ Technology, Atlanta, Ga., USA
}

\section{Key Words}

Longitudinal data - Cognitive aging · Subjective memory .

Memory performance $\cdot$ Health and Retirement Study

\begin{abstract}
Aging researchers have long been interested in understanding individuals' subjective perceptions of their own memory functioning. Previous research has shown that subjective memory ratings are partly based on memory performance but also reflect the influence of other factors, such as depressive symptoms. The aim of the present study was to examine (1) longitudinal associations between trajectories of subjective memory and memory performance, (2) variables that predict levels of and changes in subjective memory and memory performance, and (3) variables that moderate associations between these constructs. We applied a latent growth curve model to four occasions of data from 15,824 participants of the Health and Retirement Study (HRS; mean age at baseline $=64.27$ years, SD $=9.90 ; 58 \%$ women). Results revealed that latent changes in subjective memory were correlated with latent changes in memory performance $(\varphi=0.49)$, indicating that participants who reported steeper declines of subjective memory indeed showed steeper declines of memory performance over time. Three major pat-
\end{abstract}

terns of associations emerged with respect to predictors of subjective memory and subjective memory change. First, the level of memory performance showed stronger associations with age, gender, and education, whereas subjective memory was more strongly associated with subjective age and personality traits. For example, women performed better than men on the episodic memory test, but there were no gender differences in subjective memory. Also, older age was associated with steeper declines of memory performance but with less decline of subjective memory. Second, personality traits that predicted subjective memory intercepts did not predict subjective memory slopes. Third, the strength of associations between levels and slopes of subjective memory and memory performance varied as a function of gender, education, depressive symptoms, and personality traits. Conscientiousness moderated the relationship of the level of subjective memory to the level of memory performance, consistent with the hypothesis that persons high in conscientiousness more accurately monitor memory successes and failures. The results reinforce the importance of depressive symptoms as a predictor of subjective memory but also indicate that a broader perspective on the reasons why memory complaints have modest correlations with memory itself is needed.

(c) 2015 S. Karger AG, Basel

\section{KARGER 125}

(c) 2015 S. Karger AG, Basel

0304-324X/15/0613-0232\$39.50/0

E-Mail karger@karger.com

www.karger.com/ger
Gizem Hülür

Institute of Psychology

Humboldt University Berlin

Unter den Linden 6, 10099 Berlin (Germany)

E-Mail gizem.hueluer@hu-berlin.de 


\section{Introduction}

Understanding how people perceive their own memory functioning can have important implications for assessing memory dysfunction in later life. Previous research has shown that subjective memory ratings are partly based on memory performance but also independently associated with other factors, such as depressive symptoms [1]. In the present study, we examined longitudinal associations between subjective memory and memory performance and the role of sociodemographic (age, gender, education) and psychological (depressive symptoms, subjective age, personality) variables as correlates and moderators. To do so, we used 4-wave longitudinal data from the Health and Retirement Study (HRS; $n=15,824$ ), a national sample of US adults aged 50 years or older.

Some classifications of mild cognitive impairment include subjective memory complaint as a defining characteristic [2]. However, previous research has documented relatively small associations between subjective memory and memory performance. Two recent meta-analyses detected very small effect sizes, although they were positive and reliably different from $0(\mathrm{r}=0.15[3]$ and $\mathrm{r}=0.06[4])$. Moreover, Crumley et al. [4] reported that associations were moderated by a number of variables, including whether beliefs were measured as negative complaints or a positive sense of capacity. Variables other than memory, particularly depressive symptoms, correlate with subjective memory even when memory performance itself does not [5-7; for a recent review, see 1]. Hence, clinical geropsychologists have focused on the problem of distinguishing valid memory complaints from illusory concerns stemming from clinical depression or dysphoric mood [8].

The personality trait of neuroticism, which reflects a tendency to experience negative affect as well as emotional instability, is a risk factor for depression and a strong correlate of perceived psychological stress in adults [9]. Neuroticism has also been linked with subjective memory concerns [10, 11]. Pearman et al. [7] have recently found independent associations of neuroticism and diagnosed clinical depression with memory complaints in a sample of old and very old adults. Other, less frequently mentioned aspects of personality (e.g., conscientiousness) and self-esteem also correlate with adults' subjective memory beliefs $[10,12]$. For example, Hultsch et al. [13] found that the Jackson Personality Scales of Energy and Endurance correlated roughly $(r=0.3)$ with a measure of memory self-efficacy, suggesting that individuals who generally maintain high levels of energetic arousal (similar to extraversion) report better subjective memory. Lit-

Correlates of Subjective Memory the is known about associations of the other Big Five personality traits with subjective memory [but see 10]. The focus of the field on depressive symptoms as the key correlate of subjective memory may have obscured the existence of a broader set of correlates, especially personality traits, of memory self-concept in adulthood.

Although individual differences in subjective memory tend to be relatively stable in the old age, there is measureable change in subjective memory in long-term longitudinal data on older adults [14]. Recent studies using longitudinal growth curve models have found reliable latent variable correlations of change slopes for subjective memory and memory performance (i.e., $\varphi=0.24$ [15], $\varphi=0.39$ [16], $\varphi=0.44$ [17]). Perhaps longitudinal data minimize the effect of variables that influence self-concept but are unrelated to memory status, thereby enhancing the validity of reported changes in memory as an indicator of actual memory change. However, some longitudinal studies examining correlated changes have found nil to small associations $[7,14,18]$, consistent with cross-sectional metaanalyses. The reasons for discrepant longitudinal findings are not yet clear and might be based on methodological differences (e.g., nature of the population, age of the sample, measure of memory complaints vs. memory ratings).

To date, conclusions about personality-subjective memory relations have largely been based on cross-sectional data. Little is known about whether personality predicts longitudinal subjective memory change. Likewise, other variables manifest cross-sectional associations with subjective memory and could therefore predict longitudinal subjective memory change. Internalization of age stereotypes about memory as applying to oneself $[19,20]$ may affect subjective memory. Younger subjective age is known to be associated with higher memory self-efficacy [21] and fewer memory complaints [7] in older adults.

Finally, associations between subjective memory and memory performance might differ across subpopulations and have been reported to be stronger for men [15] and for individuals with higher education $[15,22]$. Hülür et al. [15] argued that men and individuals with more years of education have more experience in between-person cognitive comparisons, which has been linked to more accurate self-evaluations [for a review, see 23].

\section{The Present Study}

Typically, studies often lack the statistical power to detect small moderator effect sizes [24]. In contrast, we examined longitudinal associations between subjective 
memory, memory performance, and other correlates, especially personality, using four occasions of data from the HRS $(n=15,824)$. Based on previous research, we expected small positive associations between trajectories of subjective memory and memory performance. With respect to correlates of subjective memory, we expected those with higher education, fewer depressive symptoms, lower in neuroticism, higher in conscientiousness and lower subjective age to report higher levels of subjective memory. We expected subjective age and personality traits to be more closely related to subjective memory than to memory performance. Furthermore, we expected men and those with higher education to show stronger associations between levels of subjective memory and memory performance and explored whether these variables moderated the longitudinal slope-slope correlations.

\section{Methods}

We used longitudinal data from the HRS. Descriptions of participants, variables, and procedures are given in detail in McArdle et al. [25] and in Hülür et al. [26]. Specific details relevant to the present study are presented below.

\section{Participants and Procedures}

The HRS started in 1992 with a nationally representative probability sample of households in the United States where at least one household member was a non-institutionalized individual aged $\geq 50$ years. Data was collected every second year since 1992 and new 'refresher' cohorts were added every 6 years. Personality and subjective age were assessed in the psychosocial questionnaire of the HRS [27] starting in 2006 (personality) and 2008 (subjective age). Participants were administered the psychosocial questionnaire in alternate waves.

We used data from 15,824 participants who provided (a) at least one wave of data on subjective memory and memory performance when they were $\geq 50$ years old, and (b) information on correlates (age, gender, years of education, at least one observation of depressive symptoms, each personality trait, and subjective age). In total, we used longitudinal data that was collected on up to four measurement occasions over up to 6 years $(2006,2008,2010$, and 2012). At their first wave, participants (58\% women) were on average 64.27 years old (standard deviation, $\mathrm{SD}=9.90$, range $=50-98$ years) and had obtained 12.84 years of formal education $(\mathrm{SD}=$ 3.03).

\section{Measures}

Subjective Memory

Subjective memory was measured at each occasion with the item 'How would you rate your memory at the present time? Would you say it is excellent, very good, good, fair, or poor?', with responses provided on a 0 (poor) to 4 (excellent) scale [28]. The subjective memory item was administered prior to the test of episodic memory.
Memory Performance

Episodic memory performance was measured at each wave with tests of immediate and delayed free recall [28]. A list of 10 nouns was presented to the participants, who were asked to recall as many words as possible (a) immediately after presentation and (b) after a delay of approximately $5 \mathrm{~min}$. Tests of immediate and delayed recall were scored as the proportion of correctly remembered words and then averaged to create a single index. Higher scores indicate better memory.

\section{Correlates}

Gender, education, average age, depressive symptoms, personality, and subjective age were included as correlates in our models. Gender was a time-invariant dichotomous variable. $E d u$ cation was a time-invariant variable indicating the number of years spent in formal schooling. Age was calculated as the difference between an individual's birth year and the year of the assessment at each occasion. Average age was calculated as the mean of all available ages for each participant. Depressive symptoms were measured at all waves as the sum of responses to eight items from the Center for Epidemiologic Studies Depression Scale (CES-D) [29]. Participants indicated whether they had experienced $(0=$ no, 1 = yes) a total of eight depressive symptoms (e.g., felt depressed, everything was an effort) during the past week [30]. Depressive symptoms were averaged across all available waves for each participant.

Personality traits were measured with items derived from the International Personality Item Pool [31] as implemented in the Midlife in the United States (MIDUS) study [32]. Neuroticism was measured with 4 items (moody, worrying, nervous; reverse-coded: calm); conscientiousness (organized, responsible, hardworking, thorough; reverse-coded: careless), extraversion (outgoing, friendly, lively, active, talkative), and agreeableness (helpful, warm, caring, softhearted, sympathetic) were measured with 5 items each, and openness was measured with 7 items (creative, imaginative, intelligent, curious, broad-minded, sophisticated, adventurous). Participants indicated how well each item described them on a scale from 0 (not at all) to 3 (a lot). Zimprich et al. [32] showed that these item factors have strict measurement invariance in the MIDUS data. Items measuring each personality trait were averaged to generate personality variables ranging from 0 to 3 .

Subjective age was assessed by one item, with participants responding to the question 'What age do you feel?' [27]. Subjective age responses $<0$ or $>120$ - comprising only $0.10 \%$ of all responses - were considered invalid and treated as missing data.

\section{Data Preparation}

The schedule of administration varied for the specific variables. Subjective memory, memory performance, and depressive symptoms were measured at all four waves from 2006 to 2012. Participants were administered the personality measure either in 2006 and 2010 or in 2008 and 2012. Subjective age was measured at the waves 2008 and 2012 or in 2010 . Our analysis focused on aggregate associations of depressive symptoms, personality, and subjective age with level and slope of memory and subjective memory. Hence, depressive symptoms, the personality scales, and subjective age were averaged across all available waves for each participant and used as predictors of the memory and subjective memory variables. Subjective memory and memory performance were converted into a T-score metric $($ mean $=50, \mathrm{SD}=10)$ using baseline sta- 
Table 1. Descriptive statistics and intercorrelations of subjective memory, memory performance, and correlates

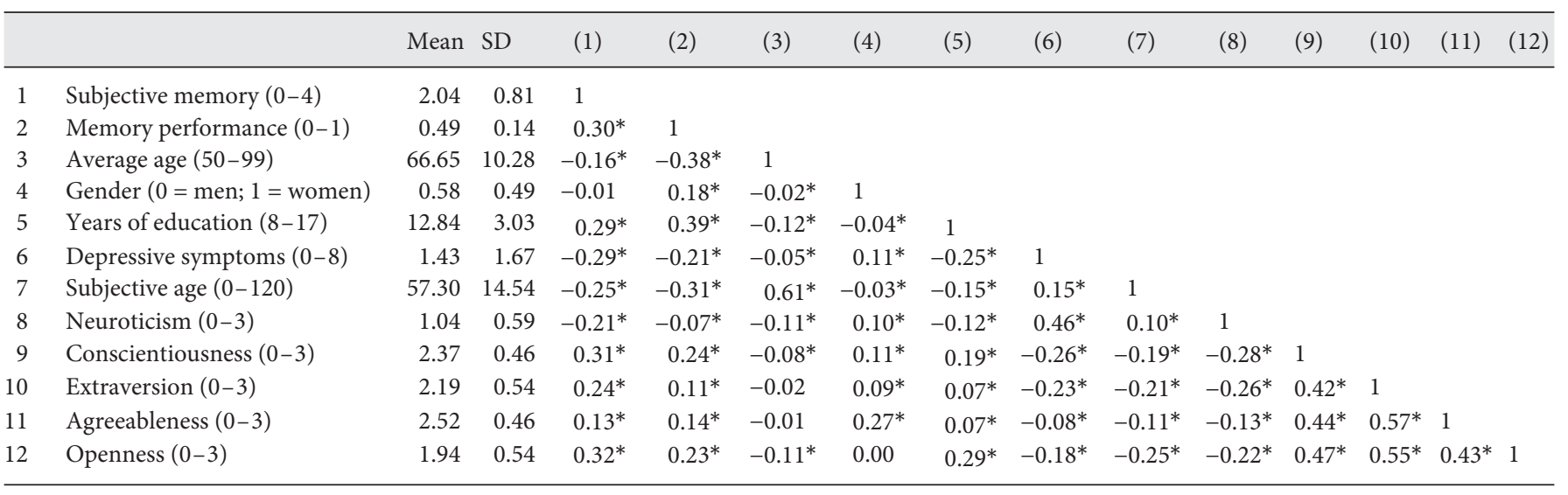

$\mathrm{n}=15,824$ participants. ${ }^{*} \mathrm{p}<0.01$.

tistics of our sample. Table 1 shows the descriptive statistics for and correlations among time-invariant and aggregated time-varying variables.

\section{Data Analysis}

To evaluate longitudinal associations between trajectories of subjective memory and memory performance over time, we estimated an occasion-based latent growth curve model with Mplus [33] using the Full Information Maximum Likelihood estimation. Latent intercepts and slopes were centered at baseline. To examine whether our set of correlates predicted trajectories of subjective memory and memory performance, latent factors indicating level and change were regressed on the correlates centered at sample means. Furthermore, we controlled for retest effects. Since we did not include data from waves prior to 2006, only $23 \%$ of our sample had no retest at baseline. The remaining $77 \%$ of the sample had already been tested with the episodic memory test in prior waves not included in the present study. The latent factor reflecting the level of memory performance at baseline was regressed on a binary variable $(0=$ no retest, $1=$ retest $)$ that was centered at the sample mean. Given the large sample size, we set the type I criterion to $\mathrm{p}<0.01$. We note that even with this criterion some very small associations were able to reach significance (table 1).

In a further step, we evaluated the moderating role of our correlates for associations between subjective memory and memory performance. The latent factor reflecting the level of subjective memory was regressed on (a) the latent factor reflecting the level of memory performance, and (b) on the interaction between the latent level factor of memory performance and each correlate. Likewise, the latent factor reflecting the slope of subjective memory was regressed on (a) the latent factor reflecting the slope of memory performance, and (b) on the interaction between the latent slope factor of memory performance and each correlate. The interaction terms between the correlates and the latent factors were modeled using the XWITH function of MPlus. To center the latent predictor variables reflecting level of and change in memory performance, manifest indicators of memory performance were centered at their own means.

\section{Results}

\section{Longitudinal Associations between Subjective Memory and Memory Performance}

Findings from the latent growth curve model are shown in table 2 (follow-up analyses including immediate or delayed recall scores as indicators of memory performance revealed the same pattern of findings). The model fit the data very well $(\mathrm{CFI}=0.99$; $\mathrm{RMSEA}=0.02$; SRMR $=0.01)$. On average, subjective memory decreased slightly by -0.33 per year $(3.3 \mathrm{~T}$-score units per decade), and episodic memory declined by -0.46 per year. There were reliable individual differences in levels and rates of change for both memory and subjective memory controlling for correlates. Higher levels of subjective memory were associated with higher levels of memory performance at baseline $(\varphi=0.21)$, and persons showing steeper changes in perceived memory also showed steeper declines of actual memory performance $(\varphi=0.49)^{1}$

\footnotetext{
1 Hülür et al. [15] detected intraindividual variability around the fitted longitudinal change slopes in memory and subjective memory in the original HRS 9-wave data (that did not initially measure personality). This variability generated occasion-specific residual covariances between the two variables independent of the slope-slope covariance. To check whether omitting these occasion-specific relationships would bias the results, we fitted a model that added them. There was no major improvement in fit (CFI = 0.99; RMSEA = 0.02 ; SRMR $=0.01)$, and the estimated residual correlation was small $(\mathrm{r}=$ $0.03 ; \mathrm{p}<0.01)$. The presence of these residual covariances did reduce the estimated correlation between slopes $(\varphi=0.30 ; p=0.02)$, but it did not affect regression relationships to other correlates we will report next.
} 
Table 2. Growth curve model examining trajectories of subjective memory and memory performance, and the role of correlates

\begin{tabular}{|c|c|c|c|c|}
\hline Parameter & $\begin{array}{l}\text { subjective } \\
\text { memory }\end{array}$ & $\begin{array}{l}\text { memory } \\
\text { performance }\end{array}$ & $\begin{array}{l}\text { subjective } \\
\text { memory }\end{array}$ & $\begin{array}{l}\text { memory } \\
\text { performance }\end{array}$ \\
\hline \multicolumn{5}{|l|}{ Fixed effects } \\
\hline Level at baseline & $50.00 *(0.07)$ & $50.12 *(0.06)$ & $6.33^{*}(0.05)$ & $6.92 *(0.07)$ \\
\hline Slope & $-0.33^{*}(0.01)$ & $-0.46^{*}(0.02)$ & $-0.61 *(0.04)$ & $-0.98^{*}(0.10)$ \\
\hline Gender $\rightarrow$ level & $0.13(0.15)$ & $3.30 *(0.14)$ & $0.01(0.01)$ & $0.22 *(0.01)$ \\
\hline Education $\rightarrow$ level & $0.49 *(0.02)$ & $0.86^{*}(0.02)$ & $0.19 *(0.01)$ & $0.36^{*}(0.01)$ \\
\hline Depressive symptoms $\rightarrow$ level & $-0.84 *(0.05)$ & $-0.73 *(0.05)$ & $-0.18 *(0.01)$ & $-0.17^{*}(0.01)$ \\
\hline Subjective age $\rightarrow$ level & $-0.05 *(0.01)$ & $-0.01(0.01)$ & $-0.08 *(0.01)$ & $-0.01(0.01)$ \\
\hline Neuroticism $\rightarrow$ level & $-0.40 *(0.13)$ & $0.00(0.13)$ & $-0.03 *(0.01)$ & $0.00(0.01)$ \\
\hline Age $\rightarrow$ slope & $0.01 *(<0.01)$ & $-0.03^{*}(<0.01)$ & $0.20 *(0.03)$ & $-0.59 *(0.07)$ \\
\hline Gender $\rightarrow$ slope & $0.08 *(0.03)$ & $0.00(0.03)$ & $0.07 *(0.03)$ & $0.00(0.03)$ \\
\hline Education $\rightarrow$ slope & $-0.02 *(0.01)$ & $-0.01(0.01)$ & $-0.12 *(0.03)$ & $-0.07(0.04)$ \\
\hline Depressive symptoms $\rightarrow$ slope & $0.00(0.01)$ & $-0.02(0.01)$ & $0.01(0.03)$ & $-0.06(0.04)$ \\
\hline Subjective age $\rightarrow$ slope & $-0.004^{*}(<0.01)$ & $0.00(<0.01)$ & $-0.10 *(0.03)$ & $-0.07(0.04)$ \\
\hline Neuroticism $\rightarrow$ slope & $-0.05(0.03)$ & $0.00(0.03)$ & $-0.05(0.03)$ & $0.00(0.04)$ \\
\hline Conscientiousness $\rightarrow$ slope & $-0.08(0.04)$ & $0.06(0.04)$ & $-0.07(0.03)$ & $0.06(0.04)$ \\
\hline Extraversion $\rightarrow$ slope & $-0.03(0.04)$ & $-0.08(0.04)$ & $-0.03(0.03)$ & $-0.09(0.05)$ \\
\hline Agreeableness $\rightarrow$ slope & $0.02(0.04)$ & $0.02(0.04)$ & $0.01(0.03)$ & $0.02(0.04)$ \\
\hline Openness $\rightarrow$ slope & $-0.01(0.03)$ & $0.02(0.04)$ & $-0.01(0.03)$ & $0.02(0.04)$ \\
\hline \multicolumn{5}{|l|}{ Random effects } \\
\hline \multicolumn{5}{|l|}{ Model fit } \\
\hline $\mathrm{R}^{2}$ level & $0.28^{*}(0.01)$ & $0.44^{*}(0.01)$ & & \\
\hline $\mathrm{R}^{2}$ slope & $0.06 *(0.01)$ & $0.41^{*}(0.08)$ & & \\
\hline$\chi^{2}$ (d.f.) & $406.62 *(75)$ & & & \\
\hline CFI & 0.99 & & & \\
\hline RMSEA & 0.02 & & & \\
\hline SRMR & 0.01 & & & \\
\hline
\end{tabular}

$\mathrm{n}=15,824$ participants. Subjective memory and memory performance standardized to a $\mathrm{T}$ metric $($ mean $=50, \mathrm{SD}=10)$ based on cross-sectional data of the present sample at baseline. Standard errors in parentheses. ${ }^{*} \mathrm{p}<0.01$.

\section{Correlates of Subjective Memory and Memory \\ Performance}

Table 2 reports both unstandardized and standardized regression coefficients for correlates predicting level and change in memory and subjective memory. In line with previous studies, older individuals and those reporting more depressive symptoms showed lower levels of subjective memory and memory performance. More educated persons showed higher levels of subjective memory and memory performance. Women showed higher levels 
of memory performance, but they did not report higher levels of subjective memory; in effect, women's selfratings failed to capture their superiority in episodic memory.

Of critical interest for this report, all five personality variables independently predicted initial levels of subjective memory. Aggregate zero-order correlations are shown in table 1, and partial regression coefficients are reported in table 2 . In the regression model, higher conscientiousness and higher openness were both associated with higher levels of both subjective memory and memory performance. Lower neuroticism, higher extraversion, and lower agreeableness were associated with higher levels of subjective memory but unrelated to levels of memory performance. In terms of standardized partial regression coefficients, the relationships were $>0.10$ only for conscientiousness and openness, but the personality scales were substantially intercorrelated (table 1) and the neuroticism effect controlled for depressive symptoms, a manifestation of one of its principal facets. Lower subjective age also predicted higher subjective memory.

Despite the associations of these correlates with subjective memory intercepts (stable individual differences in memory and subjective memory), none of the personality variables predicted changes in subjective memory over time, despite the large sample size. However, individuals reporting a higher subjective age also showed steeper declines of subjective memory ratings.

Age, gender, and education predicted subjective memory slopes in ways not fully consistent with changes in memory itself. Older individuals showed less steep declines of subjective memory but steeper declines of memory performance. Women showed less steep declines of subjective memory, and those with more years of formal education showed steeper declines of subjective memory. Neither of these variables predicted changes in episodic memory.

\section{Moderators of the Association between Subjective} Memory and Memory Performance

One of the major advantages of the large-sample HRS data is sufficient statistical power to evaluate moderated regression effects; in particular, we were interested in whether the correlates would moderate the relationship between subjective memory and memory performance.

Several moderators of interest were detected. The effect of the level of memory performance on the level of subjective memory $(0.16, \mathrm{SE}=0.01 ; \mathrm{p}<0.01)$ was stronger for participants with higher education (0.01 per year of education, $\mathrm{SE}<0.01 ; \mathrm{p}<0.01)$ and weaker for older participants $(-0.01, \mathrm{SE}<0.01 ; \mathrm{p}<0.01)$ and women $(-0.15$, $\mathrm{SE}=0.02 ; \mathrm{p}<0.01)$. The association between levels of subjective memory and memory performance was also stronger for more conscientious $(0.09, \mathrm{SE}=0.03 ; \mathrm{p}<0.01)$ and more agreeable participants $(0.11, \mathrm{SE}=0.03$; $\mathrm{p}<$ $0.01)$. Figure 1 illustrates the model-implied association between subjective memory and memory performance for subpopulations of participants who are 1 SD below or above the sample means in conscientiousness (fig. 1a) and agreeableness (fig. 1b).

In terms of change associations, the prediction of subjective memory change by actual memory change varied as a function of depressive symptoms. The unstandardized regression weight of the slope of memory performance on the slope of subjective memory $(1.20, \mathrm{SE}=$ 0.33 ; $p<0.01)$ was reliably stronger for participants experiencing more depressive symptoms $(0.24, \mathrm{SE}=0.06$; $\mathrm{p}<0.01)$.

\section{Discussion}

We examined longitudinal associations between subjective memory and memory performance in 4 -wave longitudinal data collected from over 15,000 older HRS participants. In line with previous research $[16,17]$, a bivariate latent growth curve model (controlling for multiple correlates) found (a) a small correlation between levels of subjective memory and levels of memory performance, and (b) a robust correlation between changes in subjective memory and memory performance that was larger than the correlation of the intercepts for these two variables. It is important to note that some longitudinal studies have found no or small slope-slope correlations $[7,14$, 18]. At present, the reason for this discrepancy is not clear. Certain methodological differences (e.g., nature of the population, age of the sample, measure of subjective memory) might be of importance.

The structural regression model also confirmed earlier findings that depressive symptoms are a robust predictor of memory complaints. However, one novel outcome in this study was the larger relation of actual memory change to change in subjective memory for persons high in depressive symptoms. This outcome could indicate that individuals experiencing dysphoria are more realistic and more concerned about their changing memory [7]. Conversely, this outcome could be framed as maintenance of a positive evaluation of one's own mem- 
Fig. 1. Illustrating average model implied between-person associations of subjective memory and memory performance for subpopulations with lower and higher levels of conscientiousness (a) and agreeableness (b). The dots are raw data from 1,000 participants. Participants with higher average memory performance also reported higher subjective memory. a More conscientious participants reported higher levels of subjective memory. The association between subjective memory and memory performance was stronger among more conscientious individuals. b Less agreeable participants reported higher levels of subjective memory. The association between subjective memory and memory performance was stronger among more agreeable individuals.

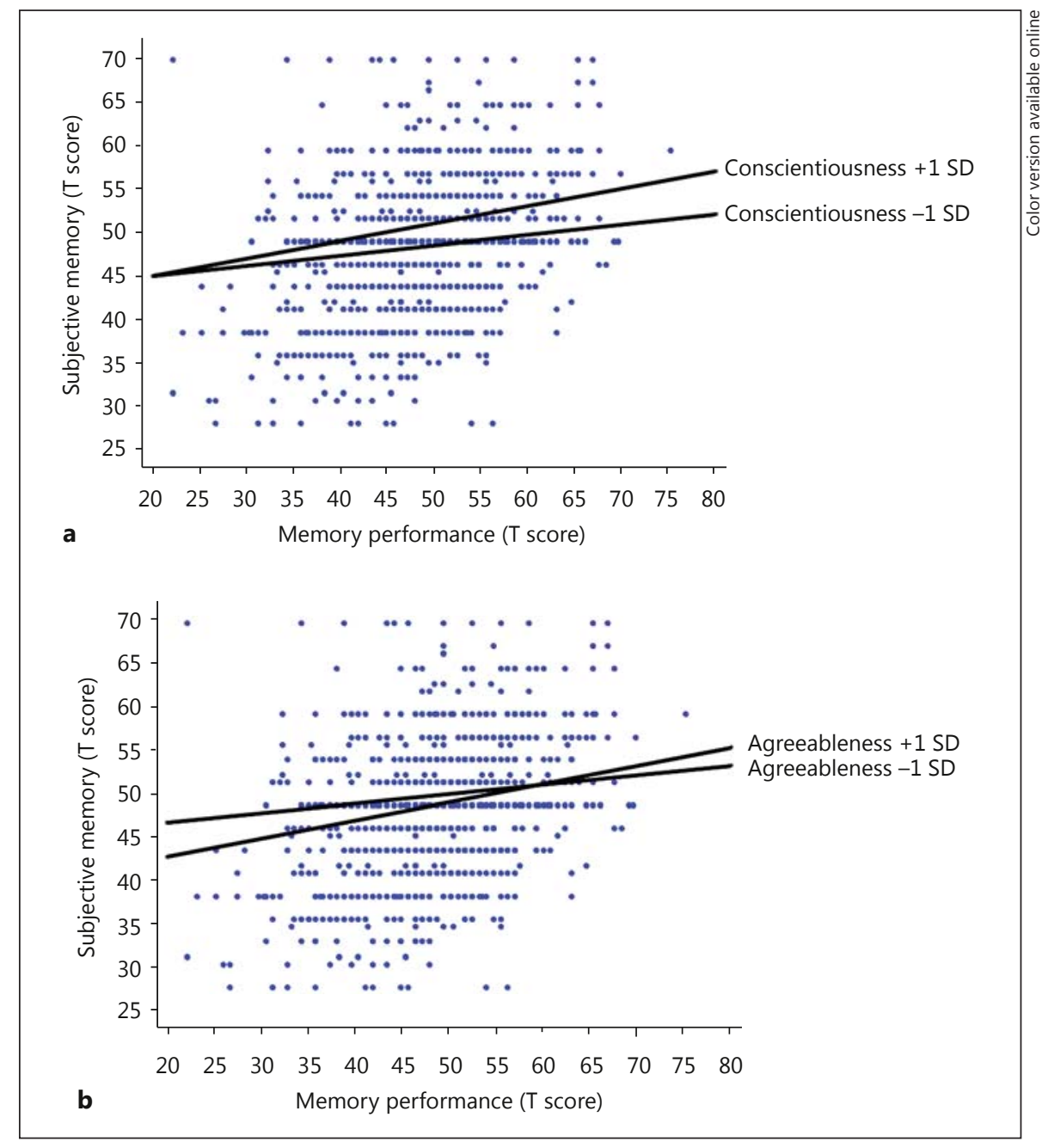

ory functioning in face of cognitive declines - a self-protective strategy that is less likely to occur for those experiencing higher levels of depressive symptoms. The effect is not consistent with the hypothesis that elevated depressive symptoms invalidly inflate subjective memory or perceptions of memory change. This finding suggests that clinicians who work with elders who show signs of possible depression should not dismiss memory complaints as merely symptomatic of the depression. Instead, the complaints could be accurate assessments of everyday memory problems.

Although the results reinforce the importance of depressive symptoms as a predictor of subjective memory, they also suggest that greater attention should be paid to aspects of personality other than neuroticism as predictors of subjective memory. In particular, conscientiousness and openness manifested robust relations to subjec- tive memory intercepts. Furthermore, conscientiousness and agreeableness moderated the relationship between memory and subjective memory intercepts. People who are more conscientious may be more likely to accurately monitor memory successes and failures on average and are perhaps also less likely to experience memory problems because they use behavioral strategies that help avoid memory errors [1].

The effects involving agreeableness were unexpected. The aggregate effect of agreeableness on subjective memory was small and negative (i.e., people who are more assertive, less nurturing, more combative, and less compliant report higher levels of subjective memory). The moderated regression effect indicated that the relationship of subjective memory to memory was stronger for agreeable persons. These patterns cannot be attributed to known gender differences in agreeableness (table 1), which were 
statistically controlled in the analysis. Perhaps individuals high in agreeableness are more accepting of their own memory failures, especially in social contexts, thereby generating a stronger relationship between subjective memory and memory. However, too little is known about the effect to draw firm conclusions.

In contrast to findings with subjective memory intercepts, personality variables did not predict changes in subjective memory over time. Similar results have been previously reported for neuroticism [7], but this study extends this conclusion to all Big Five personality factors in a study that has ample power to detect individual differences in change. Thus, these personality variables have long-term stable relations to subjective memory but do not affect subjective memory change in the old age.

The independent relation of subjective age to subjective memory intercepts, controlling for personality, is consistent with other recent cross-sectional findings [7, 21]. However, the present results showed that subjective age was also related to subjective memory slopes. Certainly the relationship warrants further investigation to ferret out its basis. On the one hand, beliefs about aging and memory may influence perceived memory; on the other hand, experiencing what are perceived to be consequential memory failures may activate aging stereotypes and lead one to 'feel older'.

The overall pattern of results reinforces the idea that subjective memory and memory, while correlated with one another, are distinct constructs. First, although older participants showed steeper declines of memory performance, they showed less steep declines of subjective memory as compared to younger participants [34]. This finding suggests that older adults may use different standards for evaluating their own memory functioning. As everyday memory problems become more common with advancing age, older adults may shift their standards or expectations for base rates of memory failures $[7,15]$. Older adults may also be less concerned with memory aging as compared to middle-aged adults [35], consistent with the age-moderation effect found in this study. Older adults also give disproportionately positive subjective health assessments, relative to the degree of objective health problems [36]. Second, subjective age and personality traits were more closely related to subjective memory than they were to memory performance. Third, the strength of associations differed across subpopulations. In addition to the moderator effects already noted, men (relative to women) and individuals with higher education showed stronger associations between levels of subjective memory and memory performance in line with previous findings $[15,22]$.

Correlates of Subjective Memory

\section{Limitations and Outlook}

We note several limitations of our study. First, the HRS surveys a wide variety of constructs with brief assessments. Although our study mostly replicated findings from previous studies using more comprehensive measures, future research should replicate some of our findings with broader measures of key constructs. In particular, the adjectives selected for the brief measures of personality in the HRS do not necessarily span all the facets that comprise higher-order factors. In the case of neuroticism, for instance, the selected adjectives draw primarily on the anxiety facet of the construct.

Second, our study included participants from a national sample of the US population and did not target a clinical sample. Further studies with clinical populations [37] are necessary to evaluate the utility of subjective ratings in clinical settings. The association between subjective memory and memory performance might be weaker or even nonexistent among those with mild cognitive impairment $[38,39]$ and dementia $[39,40]$, given concerns about anosognosia in impaired populations. An older adult presenting to a clinician with memory complaints may be depressed and should be evaluated for it, but our findings of correlated changes in subjective and episodic memory controlling depression and personality suggest that a neuropsychological assessment should also be considered to test for memory impairments. Third, measures of personality and subjective age in the HRS were only available for up to two occasions and even then at different measurement schedules. Thus, it was impractical to examine how changes in these variables might covary with changes in subjective memory and memory performance, an issue which can be addressed after future psychosocial data collections in the HRS.

\section{Conclusions}

Our study indicates that subjective memory ratings are associated with factors other than memory itself, including education, personality, and subjective age. Furthermore, the strength of associations between subjective memory and memory performance differed across subpopulations and interacted with agreeableness, conscientiousness and depressive symptoms. However, changes in subjective memory were less aligned with these variables and correlated more strongly with actual memory change. Subjective memory is a complex construct that is influenced by multiple variables. Clinicians working with old- 
er adults should keep in mind that subjective memory complaints could arise from multiple sources. Taking the time to understand the contexts, emotions, and etiology behind a self-reported memory complaint should be an important part of their assessment.

\section{Acknowledgment}

The HRS was supported by a cooperative agreement (Grant U01 AG09740) between the NIA and the University of Michigan. The content is solely the responsibility of the authors and does not necessarily represent the official views of the funding agencies.

\section{References}

1 Hertzog C, Pearman AM: Memory complaints in adulthood and old age; in Perfect TJ, Lindsay DS (eds): Handbook of Applied Memory. London, Sage, 2014, pp 423-443.

- 2 Petersen RC, Smith GE, Waring SC, Ivnik RJ, Tangalos EG, Kokmen E: Mild cognitive impairment: clinical characterization and outcome. Arch Neurol 1999;56:303-308.

-3 Beaudoin M, Desrichard O: Are memory selfefficacy and memory performance related? A meta-analysis. Psychol Bull 2011;137:211-241.

4 Crumley JJ, Stetler CA, Horhota M: Examining the relationship between subjective and objective memory performance in older adults: a meta-analysis. Psychol Aging 2014; 29:250-263.

5 Crane MK, Bogner HR, Brown GK, Gallo JJ: The link between depressive symptoms, negative cognitive bias and memory complaints in older adults. Aging Ment Health 2007;11: 708-715.

-6 Kahn RL, Zarit SH, Hilbert NM, Niederehe GA: Memory complaint and impairment in the aged: The effect of depression and altered brain function. Arch Gen Psychiatry 1975;32: 1569-1573.

7 Pearman A, Hertzog C, Gerstorf D: Little evidence for links between memory complaints and memory performance in very old age: longitudinal analyses from the Berlin Aging Study. Psychol Aging 2014;29:828-842.

8 Niederehe G, Yoder C: Metamemory perceptions in depressions of young and older adults. J Nerv Ment Dis 1989;177:4-14.

-9 Morgan ES, Umberson K, Hertzog C: Construct validation of self-reported stress scales. Psychol Assess 2014;26:90-99.

10 Pearman A, Storandt M: Predictors of subjective memory in older adults. J Gerontol B Psychol Sci Soc Sci 2004;59B:P4-P6.

11 Reid LM, MacLullich AJ: Subjective memory complaints and cognitive impairment in older people. Dement Geriatr Cogn 2006;22:471-485.

-12 Slavin MJ, Brodaty H, Kochan NA, Crawford JD, Trollor JN, Draper B, et al: Prevalence and predictors of 'subjective cognitive complaints' in the Sydney Memory and Ageing Study. Am J Geriatr Psychiatry 2010;18:701-710.

13 Hultsch DF, Hertzog C, Dixon RA, Davidson $\mathrm{H}$ : Memory self-knowledge and self-efficacy in the aged; in Howe ML, Brainerd CJ (eds): Cognitive Development in Adulthood: Progress in Cognitive Development Research. New York, Springer, 1988, pp 65-92.

14 Lane CJ, Zelinski EM: Longitudinal hierarchical linear models of the memory functioning questionnaire. Psychol Aging 2003;18:38-53.
15 Hülür G, Hertzog C, Pearman A, Ram N, Gerstorf D: Longitudinal associations of subjective memory with memory performance and depressive symptoms: between-person and within-person perspectives. Psychol Aging 2014;29:814-827.

16 Mascherek A, Zimprich D: Correlated change in memory complaints and memory performance across 12 years. Psychol Aging 2011; 26:884-889.

17 Parisi JM, Gross AL, Rebok GW, Saczynski JS, Crowe M, Cook SE, et al: Modeling change in memory performance and memory perceptions: findings from the ACTIVE study. Psychol Aging 2011;26:518-524.

18 McDonald-Miszczak L, Hertzog C, Hultsch DF: Stability and accuracy of metamemory in adulthood and aging: a longitudinal analysis. Psychol Aging 1995;10:553-564.

19 Hummert ML: Age stereotypes and aging; in Schaie KW, Willis SL (eds): Handbook of the Psychology of Aging. San Diego, Elsevier Academic Press, 2011, pp 249-262.

20 Weiss D, Freund AM: Still young at heart: negative age-related information motivates distancing from same-aged people. Psychol Aging 2012;27:173-180.

21 Stephan Y, Caudroit J, Chalabaev A: Subjective health and memory self-efficacy as mediators in the relation between subjective age and life satisfaction among older adults. Aging Ment Health 2011;15:428-436.

22 Zelinski EM, Burnight KP, Lane CJ: The relationship between subjective and objective memory in the oldest old: comparisons of findings from a representative and a convenience sample. J Aging Health 2001;13:248-266.

23 Mabe PA, West SG: Validity of self-evaluation of ability: A review and meta-analysis. J Appl Psychol 1982;67:280-296.

24 McClelland GH, Judd CM: Statistical difficulties of detecting interactions and moderator effects. Psychol Bull 1993;114:373-390.

25 McArdle JJ, Fisher GG, Kadlec KM: Latent variable analyses of age trends from the Health and Retirement Study, 1992-2004. Psychol Aging 2007:22;525-545.

26 Hülür G, Infurna FJ, Ram N, Gerstorf D: Cohorts based on decade of death: no evidence for secular trends favoring later cohorts in cognitive aging and terminal decline in the AHEAD study. Psychol Aging 2013;28:115-127.

27 Smith J, Fisher G, Ryan L, Clarke P, House J, Weir D: Psychosocial and Lifestyle Questionnaire 2006-2010. Documentation Report Core Section LB, Ann Arbor, Survey Research Center, University of Michigan.
28 Herzog AR, Wallace RB: Measures of cognitive functioning in the AHEAD Study. J Gerontol B Psychol Sci Soc Sci 1997;52B:37-48.

29 Radloff LS: The CES-D scale: A self-report depression scale for research in the general population. Appl Psychol Meas 1977;1:385-401.

- 30 Gerstorf D, Hoppmann CA, Kadlec KM, McArdle JJ: Memory and depressive symptoms are dynamically linked among married couples: Longitudinal evidence from the AHEAD Study. Dev Psychol 2009;45:1595-1610.

31 Goldberg LR, Johnson JA, Eber HW, Hogan $\mathrm{R}$, Ashton MC, Cloninger CR, et al: The international personality item pool and the future of public-domain personality measures. J Res Pers 2006;40:84-96.

32 Zimprich D, Allemand M, Lachman ME: Factorial structure and age-related psychometrics of the MIDUS personality adjective items across the life span. Psychol Assess 2012;24: 173-186.

33 Muthén LK, Muthén BO: Mplus User's Guide, ed 7. Los Angeles, Muthén \& Muthén, 1998-2012.

34 Merema M, Speelman C, Kaczmarek E, Foster $\mathrm{J}$ : Age and premorbid intelligence suppress complaint-performance congruency in raw score measures of memory. Int Psychogeriatr 2012;24:397-405.

-35 Rabbitt P, Maylor E, McInnes L, Bent N Moore B: What goods can self-assessment questionnaires deliver for cognitive gerontology? Appl Cogn Psychol 1995;9:S127-S152.

36 Idler EL: Age differences in self-assessments of health: age changes, cohort differences, or survivorship? J Gerontol 1993;48:S289-S300.

- 37 Lehrner J, Moser D, Klug S, Gleiss A, Auff E, Pirkera W, Pusswald G: Subjective memory complaints, depressive symptoms and cognition in Parkinson's disease patients. Eur J Neurol 2014;21:1276-1277.

38 Chung JC, Man DK: Self-appraised, informantreported, and objective memory and cognitive function in mild cognitive impairment. Dement Geriatr Cogn 2009;27:187-193.

-39 Farias S, Mungas D, Jagust W: Degree of discrepancy between self and other-reported everyday functioning by cognitive status: dementia, mild cognitive impairment, and healthy elders. Int J Geriatr Psychiatry 2005; 20:827-834.

40 Buckley T, Norton MC, Deberard M, WelshBohmer KA, Tschanz JT: A brief metacognition questionnaire for the elderly: comparison with cognitive performance and informant ratings. The Cache County Study. Int Geriatr Psychiatry 2010;25:739-747. 